bioRxiv preprint doi: https://doi.org/10.1101/2021.07.02.450853; this version posted November 2, 2021. The copyright holder for this preprint (which was not certified by peer review) is the author/funder. All rights reserved. No reuse allowed without permission.

\title{
Eukaryotic stress induced mutagenesis is limited by a local control of Translesion Synthesis
}

\author{
Katarzyna H. Masłowska1 ${ }^{1}$, Florencia Villafañez ${ }^{1}$, Luisa Laureti¹, Shigenori Iwai², Vincent Pagès ${ }^{1 *}$
}

\author{
${ }^{1}$ Cancer Research Center of Marseille: Team DNA Damage and Genome Instability | CNRS, Aix Marseille Univ, Inserm, \\ Institut Paoli-Calmettes, Marseille, France \\ ${ }^{2}$ Graduate School of Engineering Science, Osaka University, Osaka, Japan \\ *vincent.pages@cnrs.fr
}

\begin{abstract}
:
The DNA Damage Response (DDR) preserves the genetic integrity of the cell by sensing and repairing damages after a genotoxic stress. Translesion Synthesis (TLS), an error-prone DNA damage tolerance pathway, is controlled by PCNA ubiquitination. In this report, we raise the question whether TLS is controlled locally, or globally. Using a recently developed method that allows to follow the bypass of a single lesion inserted into the yeast genome, we show that: i) TLS is controlled locally at each individual lesion by PCNA ubiquitination, ii) a single lesion is enough to induce PCNA ubiquitination, and iii) PCNA ubiquitination is an imperative requirement for TLS to occur. More importantly, we show that global PCNA ubiquitination that follows a genotoxic stress does not increase TLS at individual lesions. We conclude that unlike the SOS response in bacteria, the eukaryotic DDR does not promote TLS and mutagenesis.
\end{abstract}

\section{INTRODUCTION}

Accurate DNA replication is essential for genome stability. Since DNA is constantly insulted by endogenous and exogenous DNA-damaging agents, organisms have evolved several mechanisms to deal with DNA damage. The DNA damage response (DDR) includes cell cycle arrest, lesion repair, and lesion tolerance (1). Numerous repair systems remove various modifications from DNA in an error-free manner (2). However, despite their efficient action, it is inevitable that some lesions might be present during replication. Most DNA damage impedes DNA synthesis by highfidelity replicative DNA polymerases. Therefore, to complete replication and maintain cell survival in the presence of residual DNA damage, cells have evolved two lesion tolerance mechanisms: Damage Avoidance (DA) and Translesion Synthesis (TLS). Damage avoidance (also named strand switch, copy choice or homology directed gap repair) is a pathway relying on the information of the newly replicated sister chromatid to circumvent the lesion in an error-free manner ((3) (4), reviewed in (5)). Translesion synthesis is a potentially mutagenic pathway that employs specialized DNA polymerases able to insert a nucleotide directly opposite the lesion (reviewed in (6) and (7)).

How the DDR controls mutagenesis has been widely studied in prokaryotic cells: the SOS response, by increasing the expression level of TLS polymerases in response to a genotoxic stress, greatly contributes to mutagenesis and therefore to the adaptive response to environmental stress (8). A good example of this phenomenon is the importance of the SOS response in resistance to antibiotics (9). Moreover, experiments involving the study of a single lesion have shown that the pre-induction of the SOS system by a genotoxic stress greatly increases the level of TLS and mutagenesis at the studied lesion (10) (11). Hence, in bacteria, two factors contribute to the increase in mutagenesis in response to a genotoxic stress: i) the number of lesions (the higher the number of lesions, the higher the probability to generate a mutation); ii) the increased level of TLS polymerases in response to the induction of SOS (the more TLS polymerases, the higher probability to bypass the lesion by TLS). Thus, the SOS response is a global response that favors TLS and mutagenesis.

The eukaryotic DDR includes mostly posttranslational modifications such as phosphorylation and ubiquitination (12). Damage-induced transcriptional regulation is less common but has also been reported (13). PCNA ubiquitination regulates lesion tolerance in response to DNA damage. After the replication fork encounters a DNA lesion, PCNA stalls at the lesion site, and single-stranded DNA (ssDNA) is generated downstream of the lesion. The formation of RPA protein-coated SsDNA leads to the recruitment of the Rad6-Rad18 complex and the subsequent monoubiquitination of PCNA at lysine K164 (14) (15) (16). This mono-ubiquitination can be further extended by Rad5 and Ubc13-Mms2, through the formation of K63-linked ubiquitin chains (17) (18). It is well established that in eukaryotic cells, PCNA mono-ubiquitination stimulates TLS, while PCNA polyubiquitination triggers DA (reviewed in (19)).

Forward mutagenesis assays have shown that the mutation frequency rapidly increases with the amount 
of genotoxic stress inflicted to the cell. While it is expected that the level of mutagenesis increases with the number of lesions (more lesions lead to more mutations), it is not known if an additional regulatory mechanism also contributes to the increase in mutagenesis in eukaryotic cells. Traditional bulk approaches do not allow to determine if the mutagenesis level is solely correlated to the number of lesions, or if a more global DNA damage response also favors mutagenesis.

As PCNA ubiquitination controls TLS, and PCNA ubiquitination increases in response to genotoxic stress, it appears intuitive that such global response exists: the more PCNA is mono-ubiquitinated, the more TLS will be used by the cell. However, the existence of such global response has never been demonstrated.

In this study, we set out to determine whether the level of TLS is regulated solely at the local level, or if the amount of damage present in the cell could favor TLS in a more global manner. We have recently devised a method to introduce a single lesion in the genome of a yeast cell (Figure 1A) (20). Such approach allows to dissect the regulation of the tolerance mechanisms in different genetic backgrounds as well as in different conditions of external stress for the cell. We have used our assay to determine whether the ratio between TLS and DA at the level of a single lesion is modulated by increasing level of genotoxic stress resulting in global DNA damage response and abundant PCNA ubiquitination.

\section{RESULTS}

\section{- PCNA ubiquitination occurs locally at a single lesion}

The assay we have previously described (20) is based on a non-replicative plasmid containing a single lesion which is stably integrated into a specific site of the yeast genome. The precise integration of the plasmid DNA into the chromosome restores a functional lacZ gene, enabling the phenotypical detection of TLS and DA events (as blue and white colonies on X-gal indicator media). In order to study tolerance events, we inactivated the following repair mechanisms: nucleotide excision repair (rad14), photolyase (phr1), and mismatch repair system (msh2). Tolerance events are calculated as the ratio of colonies resulting from the integration of the damaged vector versus the nondamaged vector.

Using this method, we have introduced a N2dG-AAF (N2-dG-Acetylaminofluorene) adduct in the genome of cells deficient for repair mechanisms, but proficient for lesion tolerance. 2-Acetylaminofluorene is a potent carcinogen known to produce liver tumors in rats (26). We observe for this lesion a TLS level of $18 \%$ that relies almost exclusively on the TLS polymerases Rev1 and Pol $\zeta$ (Figure 1B). We also observed a reduction of TLS in

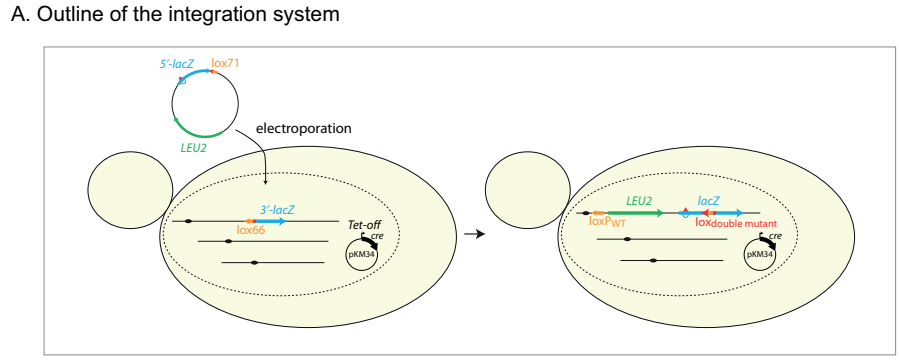

B. Requirement of PCNA ubiquitination for TLS

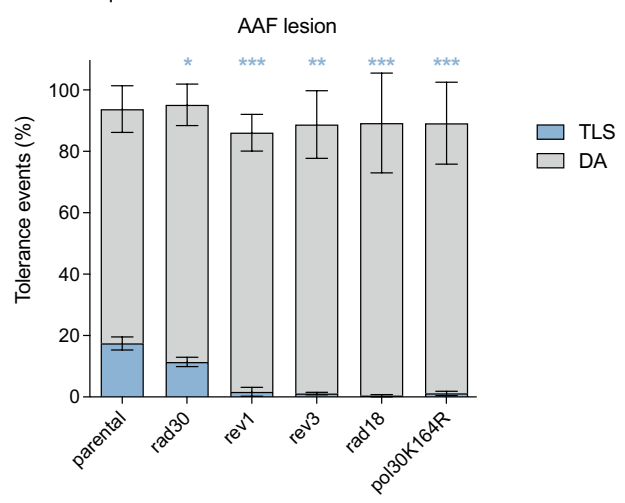

Figure 1: Requirement of PCNA ubiquitination for TLS. (A) Outline of the integration system: A non-replicative plasmid containing a single lesion is integrated into a yeast chromosome using a Cre/lox site-specific recombination. The integrative vector carrying a selection marker (LEU2) and the $5^{\prime}$-end of the lacz reporter gene containing a single lesion is introduced into a specific locus of the chromosome with the $3^{\prime}$-end of lacz. The precise integration of the plasmid DNA into the chromosome restores a functional lacZ gene, enabling the phenotypical detection of TLS and DA events (as blue and white colonies on X-gal indicator media). (B) Bypass of the N2dG-AAF lesion in strains deficient in TLS polymerases (Rev1, Pol n/ rad30, Pol $/ /$ rev3) and strains deficient in PCNA ubiquitination (rad18 and pol30K164R mutant).

the rad30 mutant showing that Pol $\eta$ is also involved in the bypass of this lesion as previously shown (27). Indeed, it has been previously suggested that Pol $\eta$ can participate to the insertion step at the N2dG-AAF lesion, while $\mathrm{Pol} \zeta$ is required for the extension step (27).

We can note that the level of TLS is highly dependent on the type of lesion as we previously measured $55 \%$ of TLS for the cis-syn TT dimer lesion (cyclobutane pyrimidine dimer) and 5\% of TLS for the (6-4)TT photoproduct lesion (thymine-thymine pyrimidine(6-4)pyrimidone photoproduct) (20). Replication through all of these lesions relies exclusively on TLS polymerases as the inactivation of rad30, rev3 and/or rev1 abolishes TLS (20).

We then introduced the N2dG-AAF lesion in cells that were unable to ubiquitinylate PCNA, either in rad18 strains, or strains where the lysine 164 of PCNA was mutated into an arginine (pol30K164R) (Figure 1B). In both conditions, TLS was almost completely abolished which is consistent with the current view of PCNA ubiquitination promoting TLS. The same results were previously observed for the cis-syn TT dimer and (6-4)TT photoproduct lesions (20). Therefore, PCNA 
ubiquitination appears as an imperative requirement for TLS to occur since almost no TLS can be achieved in its absence. Given that the lesions were introduced in the cells in the absence of any other DNA damaging treatment, the presence of the single lesion is enough to generate the signal required to trigger Rad6-Rad18-mediated PCNA ubiquitination. Hence, these data indicate that PCNA ubiquitination occurs locally, and that the single patch of ssDNA generated at one lesion is enough to recruit the Rad6Rad18 complex that will ubiquitinate PCNA allowing TLS to occur at the lesion. We can note here that the DA level remains high despite the absence of PCNA ubiquitination. These events can be attributed to the "salvage recombination pathway" that has previously been described (5). This

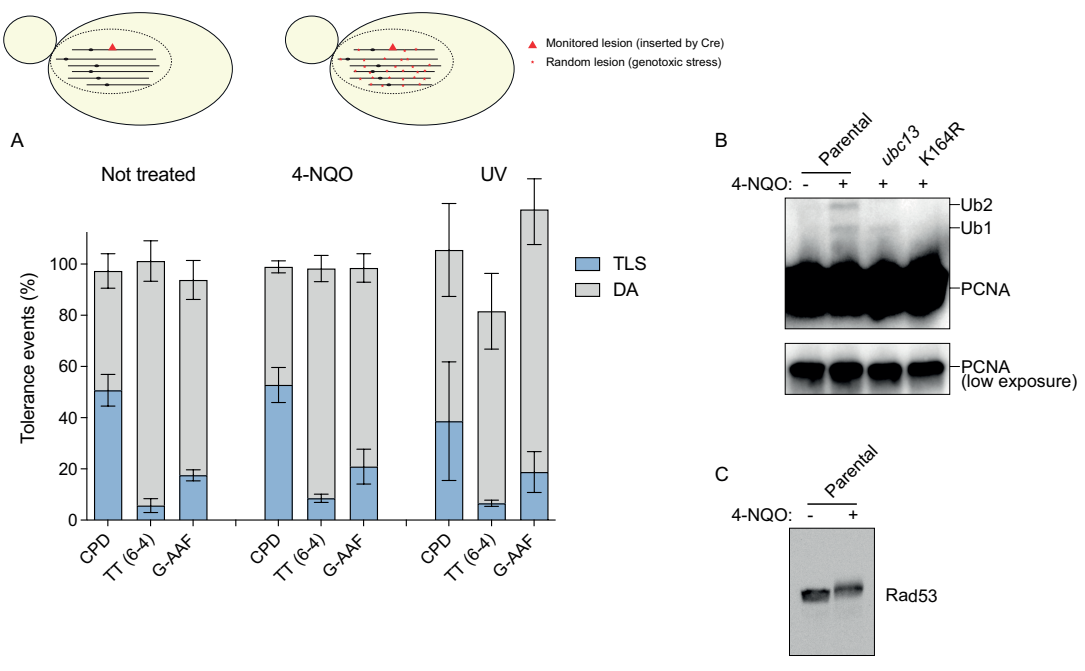

Figure 2: TLS is not modulated by a global stress response. (A) Partition of DNA Damage Tolerance events for different lesions (CPD, TT (6-4) and G-AAF) non-treated (left) and treated with 4-NQO or UV irradiation (right) prior to the integration. No significant difference was observed between the non-treated and treated conditions. (B) Western blot analysis revealing flag-tagged PCNA shows that the electroporation conditions do not induce significant level of PCNA ubiquitination. Upon treatment, two bands appear that correspond to mono- and biubiquitination of PCNA. In the pol30-K164R mutant, these two bands are no longer present since the site of ubiquitination of PCNA (Lysine 164) is absent. In the ubc13 mutant, the polyubiquitination band disappears, leaving only the monoubiquitinated version of PCNA. (C) Western blot analysis revealing Rad53 phosphorylation in response to 4-NQO treatment.

pathway allows to bypass the lesion using homologous recombination, but occurs independently of Rad6-Rad18 (28).

We then wondered if in addition to this local regulation, a more global control over PCNA ubiquitination and TLS exists. In other words, can the substantial level of PCNA ubiquitination generated by a genotoxic stress increase the use of TLS at each lesion?

\section{- Is TLS modulated by a global stress response?}

To answer this question, we treated the cells with DNA damaging agents (either 4-NQO: 4-Nitroquinoline-1oxide (29), or UV irradiation) prior to the integration of three types of lesions (cis-syn TT dimer, (6-4)TT photoproduct or N2dG-AAF). We used a dose that causes about $80 \%$ lethality. Such treatment is known to induce a strong DDR, which includes PCNA ubiquitination and Rad53 phosphorylation. Indeed, we showed by western blot that 4-NQO treatment leads to significant PCNA ubiquitination (Figure 2B) and Rad53 phosphorylation (Figure 2C). Similarly, we showed that UV-irradiation leads to PCNA ubiquitination (supplementary Figure 1 ). We also verified that the treatment to make the cells competent for electroporation and the electroporation itself were not inducing stress triggering PCNA ubiquitination. This confirms that the TLS data obtained previously (Figure 1 ) is independent of any genotoxic stress and only related to the single inserted lesion.

Having confirmed that PCNA is ubiquitinated in response to 4-NQO or UV treatment, we introduced a single lesion cis-syn TT dimer, (6-4)TT photoproduct or
N2dG-AAF) after treating the cells with 4-NQO for 30 minutes, or after UV-irradiation. No increase in TLS was observed in cells pre-treated by 4-NQO as compared to the nontreated cells. The same results were obtained with UV-irradiated cells (Figure 2A).

It appears therefore that the substantial level of PCNA ubiquitination resulting from genotoxic stress does not modulate the bypass of individual lesions. This suggests that a preexisting pool of ubiquitinated PCNA does not affect the bypass of the newly appeared lesion. Similarly, the activation of the DDR (monitored by Rad53 phosphorylation) does not modify the level of TLS at the integrated lesion. This implies that TLS is controlled at a local level, rather than by a global DDR.
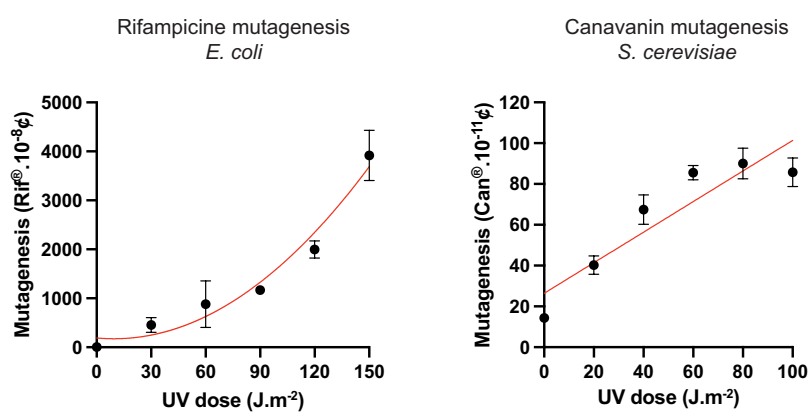

Figure 3: UV-induced mutagenesis in E. coli and S. cerevisiae. E. coli mutants were scored as rifampicin resistant colonies, $S$. cerevisiae as canavanine resistant colonies. Each point represents the average and standard deviation of at least three independent experiments. The curve in red represents the fit following a quadratic equation for the Rif mutagenesis $\left(R^{2}=0.93\right)$ or a linear fit for the Can mutagenesis $\left(R^{2}=0.82\right)$. 


\section{- Global vs. local response to DNA damage}

To confirm this hypothesis, and to avoid any bias that could be introduced by our integration system, we compared UV-induced mutagenesis in bacterial and yeast strains (Figure 3). In short, cells were UV-irradiated at different UV doses and mutants were counted as Rifampicin resistant colonies for E. coli (30), or as Canavanine resistant colonies for S. cerevisiae (31).

In E. coli, we observe a quadratic correlation between the UV dose and mutagenesis. Indeed, the number of mutations increases with both the number of lesions, and with the probability of a lesion to turn into a mutation (that also increases with the amount of lesions that induce the SOS response). Consequently, the number of mutations increases with the square of the number of lesions (quadratic correlation). This reflects the global effect of the SOS response.

On the other hand, in yeast we observe a linear correlation that even tends to reach a plateau for high doses. This shows that the genotoxic stress does not favor TLS, and mutagenesis only increases with the number of lesions. This reflects the absence of global regulation of TLS in this organism.

\section{DISCUSSION}

Our data show that a single lesion is enough to induce PCNA ubiquitination locally, and that PCNA ubiquitination is an imperative requirement for TLS to occur. More importantly, we show that a genotoxic stress leading to a significant level of PCNA ubiquitination and to the activation of the DNA damage response (Rad53 phosphorylation) has no effect on the level of TLS at individual lesions. We conclude from this data that the eukaryotic DNA damage response does not favor the increase of TLS and mutagenesis. This observation was confirmed by UV induced mutagenesis experiments showing that the mutagenic response increases only linearly with the level of damage in yeast. This response is quite different from the one in $\mathrm{E}$. coli where it has previously been shown that the level of TLS at individual lesion increases upon UV-irradiation (10), and where the mutagenic response shows a quadratic correlation with the UV dose.

The fact that the increased level of PCNA ubiquitination does not result in an increase of TLS or mutagenesis suggests that ubiquitinated PCNA is not recycled and does not stimulate TLS at other lesion sites. This implies that upon completion of a TLS patch, PCNA is either rapidly deubiquitinated, or that PCNA that is unloaded is deubiquitinated before it is recycled at another replication fork, so it will not favor TLS at another lesion site. This rapid deubiquitination could explain the low level of PCNA ubiquitination generally observed upon genotoxic stress (this study and (16)). Indeed, the level of Ub-PCNA increases when the deubiquitylases are inactivated (32).
This also implies that de novo ubiquitination occurs at each encounter with a lesion. This model is compatible with previous in vitro data that showed that PCNA remains at the lesion terminus where RPA coated SSDNA prevents its diffusion (33). In this model, a new PCNA is loaded downstream of the lesion allowing the replication to go on, while the PCNA that is maintained at the lesion site can be ubiquitinated and allows TLS to perform the gap filling reaction. Once the gap filling reaction is achieved, ubiquitinated PCNA is unloaded and will not contribute to TLS at another site.

Other events of the DDR response could affect the level of TLS besides PCNA ubiquitination. For instance, TLS polymerase Rev1 has been reported to be phosphorylated in response to a genotoxic stress (34), which was demonstrated to be important for DNA damage bypass (35). It appears from our data that such modifications are not playing a global role, but again have most likely a local effect at each lesion.

Taken together, our data show that the regulation of TLS occurs locally, and that in eukaryotic cells, there is no global response capable of increasing the level of TLS and mutagenesis in response to a genotoxic stress. The level of mutagenesis depends solely on the number of lesions present in the genome (following a linear correlation). Similar mutagenesis assays in human cells have shown the same linear response $(36,37)$. Unlike bacteria that show a quadratic increase in mutagenesis, allowing them to rapidly mutate and adapt to a stressful environment, eukaryotic cells seem to have evolved a more controlled mutagenic response to the environmental stress.

\section{MATERIAL AND METHODS}

\section{Strains and media}

All strains used in the present study are derivative of strain EMY74.7 (21) (MATa his3- $\Delta 1$ leu2-3,112 trp1- $\Delta$ ura3- $\Delta$ met25- $\Delta$ phr1- $\Delta$ rad14- $\Delta$ msh2 $\Delta:$ :hisG). Gene disruptions were achieved using PCR-mediated seamless gene deletion (22) or URAblaster (23) techniques. All strains used in the study are listed in Table 1.

\section{Integration system}

Integration of plasmids carrying cis-syn TT dimer / 6-4 (TT) / N2dG-AAF lesions (or control plasmids without lesion) and result analysis was performed as previously described (20). In experiments where cells were treated with 4-NQO, the overnight culture was inoculated into $100 \mathrm{ml}$ of yeast extract/peptone/dextrose medium (YPD) per integrated lesion to reach OD600 $=0.3$, and incubated at $30^{\circ} \mathrm{C}$ with shaking until OD600 $=0.8$. After the addition of $150 \mathrm{ng} / \mathrm{ml}$ of 4-NQO, cultures were incubated for 30 more minutes. 4-NQO was inactivated by adding an equal volume of $10 \%$ sodium thiosulfate, and then cells were further washed and processed the 
same way as untreated cultures. For UV treatment, the overnight culture was inoculated into $50 \mathrm{ml}$ of YPD per integrated lesion to reach $\mathrm{OD} 600=0.3$, and incubated at $30^{\circ} \mathrm{C}$ with shaking until $O D 600=1.6$. Cells were then harvested, resuspended in twice the initial volume of water, and treated with UV (4J.m-2) in Petri dishes (15 $\mathrm{cm} E, 25 \mathrm{ml} / \mathrm{dish})$. Cells were further washed and processed the same way as untreated cultures.

All experiments were performed in triplicate or more. Only the N2dG-AAF lesion in the UV-induced condition was done in duplicate. Graphs and statistical analysis were performed using GraphPad Prism applying unpaired t-test. Bars represent the mean value \pm s.d.

\section{Preparation of an oligonucleotide containing the (6-4) photoproduct}

A 13-mer oligonucleotide, d(GCAAGTTAACACG), purchased from Tsukuba Oligo Service (150 nmol) was dissolved in water $(7.5 \mathrm{~mL})$, and after a nitrogen purge for $5 \mathrm{~min}$, the solution was heated at $75^{\circ} \mathrm{C}$ for $5 \mathrm{~min}$ and cooled in ice. This solution was poured into a petri dish with a diameter of $8.5 \mathrm{~cm}$ and irradiated for $20 \mathrm{~min}$ on a Spectrolinker XL-1500 UV Crosslinker (Spectronics Corp.) equipped with six $15 \mathrm{~W}$ germicidal lamps (254nm, 2.2 $\mathrm{mW} . \mathrm{cm}-2)$. The reaction mixture was analyzed by reversed-phase HPLC using a Waters $\mu$ Bondasphere $\mathrm{C} 18$ $5 \mu \mathrm{m} 300 \AA$ column $\left(3.9^{\prime} 150 \mathrm{~mm}\right)$ at $50^{\circ} \mathrm{C}$ with an acetonitrile gradient of 6 to $10 \%$ during $20 \mathrm{~min}$ in $0.1 \mathrm{M}$ triethylammonium acetate $(\mathrm{pH} 7.0)$. The elution was monitored by using a Waters 2998 photodiode array detector, and the product with absorption at $325 \mathrm{~nm}$ (which is characteristic of the (6-4) photoproduct) was isolated by repeating injection of $500 \mu \mathrm{L}$. After concentration of the combined eluates, impurities detected by the second HPLC analysis using a GL Sciences Inertsil ODS-3 5um column (4.6' $250 \mathrm{~mm}$ ) with

Table 1: Strains used i, the study an acetonitrile gradient of 8 to $11 \%$ were removed. The final eluate (chromatogram shown in Supplementary Figure 2) was concentrated and co-evaporated with water. The yield determined from the absorbance at 260 $\mathrm{nm}$ was $4.5 \mathrm{nmol}$. This process is similar to the one previously described by LeClerc et al. (24).

\section{Immunoprecipitation and Western-Blot}

Cells expressing FLAG-tagged PCNA were grown and processed as for the integration of the plasmid with a lesion. After electroporation cells were left to recover during $30 \mathrm{~min}$, harvested, washed with buffer containing glycerol (1x PBS, 10\% glycerol, and $1 \mathrm{mM}$ EDTA), and frozen at $-80^{\circ} \mathrm{C}$. For protein extraction cells were resuspended in protein extraction buffer (1xPBS, $10 \%$ glycerol, 1mM EDTA, 1mM PMSF, 1x Roche cOmplete ${ }^{\text {TM }}$ Protease Inhibitor Cocktail), and lysed in a bead beater. Lysate was clarified by centrifugation, and total protein concentration was determined using Qubit Fluorometer according to manufacturer's protocol (ThermoFisher). Crude protein extract was incubated with Anti-FLAG ${ }^{\circledR}$ M2 Magnetic Beads (Merck) overnight, in an IP buffer (1xPBS, 5\% glycerol, 0.5 mM EDTA, 1mM PMSF, 1x Roche cOmplete ${ }^{\text {TM }}$ Protease Inhibitor Cocktail, $2 \mathrm{mM}$ DTT). Proteins were resolved by SDS-PAGE (BioRad $12 \%$ Mini-PROTEAN ${ }^{\circledR}$ TGX $^{\mathrm{TM}}$ Precast Protein Gels) and transferred onto PVDF membranes using Bio-Rad Trans-Blot Turbo Transfer System for Western blotting. Modified and non-modified PCNA was detected using ANTI-FLAG ${ }^{\circledR}$ M2-Peroxidase (HRP) antibody (Merck).

For Rad53 detection, protein extracts for Western blot analysis were prepared by trichloroacetic acid (TCA) precipitation, as described previously (25). Proteins were resolved by SDS-PAGE (Bio-Rad 10\% MiniPROTEAN $^{\circledR}$ TGX $^{\mathrm{TM}}$ Precast Protein Gels) and transferred onto PVDF membranes. Rad53, phosphorylated and not, was detected using anti-Rad53 polyclonal antibodies (Abcam ab104232).

\begin{tabular}{|c|c|}
\hline Strain & $\begin{array}{l}\text { Relevant Genotype } \\
\text { (All yeast strains are: MATa his3- } \Delta 1 \text { leu2-3,112 trp1- } \Delta \text { ura3- } \Delta \text { met25- } \Delta \text { rad14- } \Delta \\
\text { phr1- } \Delta \text { msh2 } \Delta:: \text { hisG) }\end{array}$ \\
\hline EVP161 & E. coli MG1655 phrB ::kan \\
\hline SC22 & rad14 phr1 \\
\hline SC53 & VI(167260-167265)::(lox66-3'lacZ-MET25/lag) \\
\hline SC55 & VI(167260-167265)::(lox66-3'lacZ-MET25/lead) \\
\hline SC82 & rev1- $\triangle \mathrm{VI}(167260-167265)::($ lox66-3'lacZ-MET25/lag) \\
\hline SC83 & rev1- $\Delta$ VI(167260-167265)::(lox66-3'lacZ-MET25/lead) \\
\hline SC86 & rad304::hisG VI(167260-167265)::(lox66-3'lacZ-MET25/lag) \\
\hline SC87 & rad304::hisG VI(167260-167265)::(lox66-3'lacZ-MET25/lead) \\
\hline SC181 & rev34::hisG VI(167260-167265)::(lox66-3'IacZ-MET25/lag) \\
\hline SC182 & rev34::hisG VI(167260-167265)::(lox66-3'lacZ-MET25/lead) \\
\hline SC203 & rad184::hisG VI(167260-167265)::(lox66-3'lacZ-MET25/lag) \\
\hline SC206 & rad184::hisG III(75494-75499)::(lox66-3'lacZ-MET25/lead) \\
\hline SC236 & pol30-K164R VI(167260-167265)::(lox66-3'lacZ-MET25/lag) \\
\hline SC237 & pol30-K164R VI(167260-167265)::(lox66-3'lacZ-MET25/lead) \\
\hline SC533 & pol30::3FLAG-kan VI (167260-167265)::(lox66-3'lacZ-MET25/lag) \\
\hline SC534 & pol30::3FLAG-kan VI (167260-167265)::(lox66-3'lacZ-MET25/lead) \\
\hline SC535 & ubc13 Pol30::3FLAG-kan VI (167260-167265)::(lox66-3'lacZ-MET25/lag) \\
\hline SC537 & pol30-K164R::3FLAG-kan VI (167260-167265)::(lox66-3'lacZ-MET25/lag) \\
\hline
\end{tabular}

Rifampicin mutagenesis assay

E. coli strain EVP161 (MG1655 phrB::kan) was grown to exponential phase in LB media, centrifugated and resuspended in $10 \mathrm{mM} \mathrm{MgSO} 4$ before UV irradiation with different UV doses (0-150 J.m-2). An aliquot of cells was plated on LB to assess survival. Cells were then diluted $1 / 20$ in LB and grown for $6 \mathrm{~h}$ before plating on LB and LB + Rif $100 \mu \mathrm{g} /$ $\mathrm{ml}$. Colonies were counted after $24 \mathrm{~h}$ on LB and $48 \mathrm{~h}$ on LB+Rif.

\section{Canavanine mutagenesis assay}

Mutagenesis experiments were 
performed as described in Unk and Daraba (2014). Briefly, yeast cultures were grown to saturation in YPD, harvested, resuspended in water (108 cells/ml), and sonicated to separate clumps of cells. Serial dilutions were plated on SD-agar plates containing canavanine for mutagenesis, and on SC-agar plates for survival. Plates were left to absorb the moisture, and irradiated with different doses of UV (0-100 J.m-2). Colonies were counted after 4 days of incubation.

\section{REFERENCES}

1. Hanawalt,P.C. (2015) Historical perspective on the DNA damage response. DNA Repair (Amst) 36, 2-7.

2. Friedberg,E.C. (2006) DNA repair and mutagenesis. Amer Society for Microbiology.

3. Hoege,C., Pfander,B., Moldovan,G.-L., Pyrowolakis,G. and Jentsch,S. (2002) RAD6-dependent DNA repair is linked to modification of PCNA by ubiquitin and SUMO. Nature 419, 135-141.

4. Branzei,D., Seki,M. and Enomoto,T. (2004) Rad18/ Rad5/Mms2-mediated polyubiquitination of PCNA is implicated in replication completion during replication stress Genes to Cells 9, 1031-1042.

5. Branzei,D. and Szakal,B. (2016) DNA damage tolerance by recombination: Molecular pathways and DNA structures. DNA Repair (Amst) 44, 68-75.

6. Prakash,S., Johnson,R.E. and Prakash,L. (2005) Eukaryotic translesion synthesis DNA polymerases: specificity of structure and function. Annual review of biochemistry 74, 317-353.

7. Waters,L.S., Minesinger,B.K., Wiltrout,M.E., D'Souza,S., Woodruff,R.V. and Walker,G.C. (2009) Eukaryotic translesion polymerases and their roles and regulation in DNA damage tolerance. Microbiology and molecular biology reviews : MMBR 73, 134-154.

8. Maslowska,K.H., Makiela-Dzbenska,K. and Fijalkowska,I.J. (2018) The SOS System: A Complex and Tightly Regulated Response to DNA Damage. Environmental and molecular mutagenesis 7, e1002244.

9. Barrett,T.C., Mok,W.W.K., Murawski,A.M. and Brynildsen,M.P. (2019) Enhanced antibiotic resistance development from fluoroquinolone persisters after a single exposure to antibiotic. Nat Commun 10, 1177.

10. Naiman,K., Philippin,G., Fuchs,R.P. and Pagès,V. (2014) Chronology in lesion tolerance gives priority to genetic variability. Proceedings of the National Academy of Sciences of the United States of America 111, 5526-5531.

11. Napolitano,R., Janel-Bintz,R., Wagner,J. and Fuchs,R.P. (2000) All three SOS-inducible DNA polymerases (Pol II, Pol IV and Pol V) are involved in induced mutagenesis. Embo J 19, 6259-6265.

12. McIntyre,J. and Woodgate,R. (2015) Regulation of translesion DNA synthesis: Posttranslational modification of lysine residues in key proteins. DNA repair 29, 166-179.

13. Fu,Y., Pastushok,L. and Xiao,W. (2008) DNA damageinduced gene expression in Saccharomyces cerevisiae. FEMS Microbiol Rev 32, 908-926.

14. Jentsch,S., McGrath,J.P. and Varshavsky,A. (1987) The yeast DNA repair gene RAD6 encodes a ubiquitinconjugating enzyme. Nature 329, 131-134.

15. Bailly,V., Lauder,S., Prakash,S. and Prakash,L. (1997) Yeast DNA repair proteins Rad6 and Rad18 form a heterodimer that has ubiquitin conjugating, DNA binding, and ATP hydrolytic activities. The Journal of biological chemistry 272, 23360-23365.

16. Davies,A.A., Huttner,D., Daigaku,Y., Chen,S. and Ulrich,H.D. (2008) Activation of ubiquitin-dependent DNA damage bypass is mediated by replication protein a Mol Cell 29, 625-636.

17. Ulrich,H.D. and Jentsch,S. (2000) Two RING finger proteins mediate cooperation between ubiquitinconjugating enzymes in DNA repair. Embo J 19, 3388-3397.

18. Parker,J.L. and Ulrich,H.D. (2009) Mechanistic analysis of PCNA poly-ubiquitylation by the ubiquitin protein ligases Rad18 and Rad5. EMBO J 28, 3657-3666.

19.Andersen,P.L., Xu,F. and Xiao,W. (2008) Eukaryotic DNA damage tolerance and translesion synthesis through covalent modifications of PCNA. Cell research 18, 162-173.

20. Masłowska,K.H., Laureti,L. and Pagès,V. (2019) iDamage: a method to integrate modified DNA into the yeast genome. Nucleic acids research 18, 563e124.

21. Johnson,R.E., Torres-Ramos,C.A., Izumi,T., Mitra,S., Prakash,S. and Prakash,L. (1998) Identification of APN2, the Saccharomyces cerevisiae homolog of the major human AP endonuclease HAP1, and its role in the repair of abasic sites. Genes Dev 12, 3137-3143.

22. Akada,R., Kitagawa,T., Kaneko,S., Toyonaga,D., Ito,S., Kakihara,Y., Hoshida,H., Morimura,S., Kondo,A. and Kida,K. (2006) PCR-mediated seamless gene deletion and marker recycling in Saccharomyces cerevisiae. Yeast (Chichester, England) 23, 399-405.

23. Alani,E., Cao,L. and Kleckner,N. (1987) A method for gene disruption that allows repeated use of URA3 selection in the construction of multiply disrupted yeast strains. Genetics 116, 541-545.

24. LeClerc,J.E., Borden,A. and Lawrence,C.W. (1991) The thymine-thymine pyrimidine-pyrimidone(6-4) ultraviolet light photoproduct is highly mutagenic and specifically induces $3 \&$ apos; thymine-to-cytosine transitions in Escherichia coli. Proceedings of the National Academy of Sciences of the United States of America 88, 9685-9689.

25. Marsella,A., Gobbini,E., Cassani,C., Tisi,R., Cannavo,E., Reginato,G., Cejka,P. and Longhese,M.P. 
bioRxiv preprint doi: https://doi.org/10.1101/2021.07.02.450853; this version posted November 2, 2021. The copyright holder for this preprint (which was not certified by peer review) is the author/funder. All rights reserved. No reuse allowed without permission.

(2021) Sae2 and Rif2 regulate MRX endonuclease activity at DNA double-strand breaks in opposite manners. Cell Rep 34, 108906.

26. Miller,E.C., Miller,J.A. and Hartmann,H.A. (1961) NHydroxy-2-acetylaminofluorene: a metabolite of 2acetylaminofluorene with increased carcinogenic activity in the rat. Cancer Res 21, 815-824.

27. Pagès,V., Bresson,A., Acharya,N., Prakash,S., Fuchs,R.P. and Prakash,L. (2008) Requirement of Rad5 for DNA polymerase zeta-dependent translesion synthesis in Saccharomyces cerevisiae. Genetics 180, 73-82.

28. Wong,R.P., García-Rodríguez,N., Zilio,N., Hanulová,M. and Ulrich,H.D. (2020) Processing of DNA Polymerase-Blocking Lesions during Genome Replication Is Spatially and Temporally Segregated from Replication Forks. Mol Cell 77, 3-16.e4.

29. Nagao,M. and Sugimura,T. (1976) Molecular biology of the carcinogen, 4-nitroquinoline 1-oxide. Adv Cancer Res 23, 131-169.

30. Foster,P.L. (1991) In vivo mutagenesis. Methods in enzymology 204, 114-125.

31. Unk,I. and Daraba,A. (2014) Measuring UV-induced Mutagenesis at the CAN1 Locus in Saccharomyces cerevisiae. Bio Protoc 4, e1272.

32. Álvarez,V., Frattini,C., Sacristán,M.P., GallegoSánchez,A., Bermejo,R. and Bueno,A. (2019) PCNA Deubiquitylases Control DNA Damage Bypass at Replication Forks. Cell reports 29, 1323-1335.e5.

33. Hedglin,M. and Benkovic,S.J. (2017) Replication Protein A Prohibits Diffusion of the PCNA Sliding Clamp along Single-Stranded DNA. Biochemistry 56, 1824-1835.

34. Sabbioneda,S., Bortolomai,I., Giannattasio,M., Plevani,P. and Muzi-Falconi,M. (2007) Yeast Rev1 is cell cycle regulated, phosphorylated in response to DNA damage and its binding to chromosomes is dependent upon MEC1. DNA repair 6, 121-127.

35. Pagès,V., Santa Maria,S.R., Prakash,L. and Prakash,S. (2009) Role of DNA damage-induced replication checkpoint in promoting lesion bypass by translesion synthesis in yeast. Genes Dev 23, 1438-1449.

36. Buglewicz,D.J., Mussallem,J.T., Haskins,A.H., Su,C., Maeda,J. and Kato,T.A. (2020) Cytotoxicity and Mutagenicity of Narrowband UVB to Mammalian Cells. Genes (Basel) 11,

37. Tang,J.Y., Hwang,B.J., Ford,J.M., Hanawalt,P.C. and Chu,G. (2000) Xeroderma pigmentosum p48 gene enhances global genomic repair and suppresses UVinduced mutagenesis. Mol Cell 5, 737-744.

\section{FUNDING:}

Fondation pour la Recherche Médicale: Equipe FRM EQU201903007797

Fondation de France 